\title{
Proteomics analysis of differentially-expressed proteins in uterus of primary dysmenorrhea mice following administration of nuangong zhitong
}

\author{
Yazhen Xie ${ }^{1,2}$, Jianqiang Qian ${ }^{2}$, Qibin $\mathrm{Lu}^{3 *}$ \\ ${ }^{1}$ Nanjing University of Chinese Medicine, Nanjing, ${ }^{2}$ Taicang Traditional Chinese Medicine Hospital Affiliated to Nanjing \\ University of Chinese Medicine, Taicang, ${ }^{3}$ Department of Gynaecology, The Affiliated Hospital of Nanjing University of Chinese \\ Medicine, Nanjing, Jiangsu, PR China
}

*For correspondence: Email: xyz8102@sina.com; Tel: +862586617141

\begin{abstract}
Purpose: To use label-free proteomic method to investigate the mechanism of action of nuanggong zhitong decoction (NZD) on primary dysmenorrhea (PD).

Methods: A mouse model of $P D$ was established through oxytocin administration. The mice were divided into control group (normal mice), model group (PD mice administered normal saline), and treatment group (mice given NZD). The serum levels of PGE2 and PGF2a in the mice were measured by ELISA. The differentially expressed proteins (DEPS) among the three groups were revealed by identifying the proteins that were up-regulated (or down-regulated) in model group and down-regulated (or up-regulated) in the treatment group. The DEPS in the three groups were identified using NanoHPLC-MS/MS, and their functions were investigated using bioinformatics analyses. The accuracy of proteomics was verified with western blot analysis.

Results: Thirty-eight up-regulated and 66 down-regulated DEPs were identified. Bioinformatics analysis revealed that the DEPs were related to immune response, signal conduction, protein binding, and metabolism. STRING analysis indicated a total of 53 DEPs have direct or indirect functional links. Western blot results revealed that levels of Stat1, Rock1, vinculin and vaveolin-1 were consistent with the results of proteomic analysis.

Conclusion: These findings provide further insights into the mechanism underlying the protective effects of NZD.
\end{abstract}

Keywords: Primary dysmenorrhea, Uterus, Nuangong zhitong decoction, Vinculin, Caveolin, Differentially expressed proteins (DEPS), Bioinformatics

This is an Open Access article that uses a fund-ing model which does not charge readers or their institutions for access and distributed under the terms of the Creative Commons Attribution License (http://creativecommons.org/licenses/by/4.0) and the Budapest Open Access Initiative (http://www.budapestopenaccessinitiative.org/read), which permit unrestricted use, distribution, and reproduction in any medium, provided the original work is properly credited.

Tropical Journal of Pharmaceutical Research is indexed by Science Citation Index (SciSearch), Scopus, International Pharmaceutical Abstract, Chemical Abstracts, Embase, Index Copernicus, EBSCO, African Index Medicus, JournalSeek, Journal Citation Reports/Science Edition, Directory of Open Access Journals (DOAJ), African Journal Online, Bioline International, Open-J-Gate and Pharmacy Abstracts

\section{INTRODUCTION}

Primary dysmenorrhea (PD) refers to recurrent menstrual cramps that are not due to other diseases. It occurs in approximately $50 \%$ of menstruating females. The pain associated with PD is extremely severe in $15 \%$ of patients, and results in psychological distress such as anxiety and depression [1]. Moreover, PD pain may be accompanied with nausea-and-vomiting, fatigue, and diarrhea [3]. Currently, the principal 
pharmacological therapies for PD include oral contraceptives and non-steroidal antiinflammatory drugs (NSAIDs). [4]. However, these drugs are associated with adverse side effects [5]. Thus, in their place, Chinese herbal medicine is used for treating PD due to its fewer adverse effects and lower degree of PD recurrence $[6,7]$.

Nuangong zhitong decoction (NZD) has been used for clinical treatment of PD for many years in China. It was developed from the traditional Chinese prescription of Wen jing decoction which has been used clinically treating dysmenorrhea for decades. [8]. Nuangong zhitong decoction (NZD) is composed of Cinnamomi ramulus, evodiamine, asarum, Radix linderae and rhizoma corydalis at the ratio of $1: 2: 1: 2: 2$. Cinnamaldehyde and cinnamic acid are the two main constituents of Cinnamomi ramulus. They have been reported to suppress oxytocininduced uterine contractions [9,10]. Evodiamine, asarum, Radix linderae and rhizoma corydalis warm the meridians so as to dissipate cold and relieve pain. However, the molecular mechanisms that underlie the analgesic effect of NZD are poorly understood. Therefore, there is need for more studies in this area.

Label-free quantitative proteomics has been employed to explore the mechanisms of medicine, including traditional Chinese Medicine (TCM) [11-14]. It emerged as a powerful approach for large-scale protein analysis with quantifying peptides and proteins with the use of a peptide's response as a quantitative measure $[15,16]$. In this study, proteomic alterations in PD mice in response to NZD treatment were investigated using Nano-HPLC-MS/MS technology.

\section{EXPERIMENTAL}

\section{Animal model of PD}

Animal experiments in this study were approved by Ethics Committee of Taicang TCM Hospital Affiliated to Nanjing University of Traditional Chinese Medicine. Female KM mice (mean weight $=25 \pm 5 \mathrm{~g}, 6-8$ weeks of age) were purchased from Cavens Laboratory Animal Co. Ltd (Changzhou, China). The mice were intragastrically administered a decoction made from gypsum, gentiana, Phellodendron chinense and Rhizoma anemarrhena, mixed in a ratio $2: 1.2: 1: 1.5$, at a dose of $4 \mathrm{~g} / \mathrm{mL}$ for 14 days to establish a mouse model of cold-type asthenia. The mice were then subcutaneously injected with estradiol benzoate injection ( $2 \mathrm{mg} / \mathrm{kg}$ ) daily for 12 days to improve the sensitivity of the mice uterine tissues to oxytocin. On the $12^{\text {th }}$ day, oxytocin (20 $\mathrm{U} / \mathrm{kg}$ ) was injected intraperitoneally to the mice to induce severe uterine contraction.

Seven days after establishment of ACT-PD model, the mice were randomly divided into 2 groups (10 mice/group) administered normal saline (model group) or NZD at a dose of 30.00 $\mathrm{g} / \mathrm{kg}$ body weight (bwt, treatment group) for another 7 days. Ten (10) healthy Balb/c mice which were intragastrically administered normal saline for 13 days (10 mL/kg bwt) served as control group. On the $13^{\text {th }}$ day, writhing reaction was induced through intraperitoneal injection of oxytocin (33 U/kg).

\section{Writhing test}

The mice were placed in a box and intraperitoneally injected with oxytocin. The number of writhes in 30 min was counted. Analgesia (A) was calculated according to Eq 1.

$A(\%)=\{(W P D-W t) / W P D\}$

where $W_{P D}$ and $W t$ are the no. of writhes in $P D$ and treatment groups, respectively

\section{Enzyme-linked immunosorbent assay (ELISA)}

Blood was collected from the retroorbital plexus of mice after administration of NZD or its bioactive components for $40 \mathrm{~min}$. The serum levels of PGE2 and PGF2 $\alpha$ were measured with ELISA kits according to the kit protocol.

\section{Sample preparation and protein digestion}

There were three samples of mouse uterus tissue in each group. After cutting them into smaller pieces, RIPA lysis buffer was added, and the tissues were mechanically homogenized using a tissue homogenizer thrice, each for 3 sec. After an incubation of $15 \mathrm{~min}$ on ice, the samples were centrifuged at $12,000 \mathrm{~g}$ for $15 \mathrm{~min}$ at $4^{\circ} \mathrm{C}$, and the supernatants were separately transferred into new Eppendorf tubes. BCA assay was used for the detection of the protein concentration of the supernatant. Proteins were diluted with $8 \mathrm{M}$ urea solution followed by a further incubation of $1 \mathrm{~h}$ at $37^{\circ} \mathrm{C}$. Thereafter, the mixture was transferred into $10 \mathrm{~K}$ Microcon centrifugal filter unit (Millipore, Billerica, MA). The samples were centrifuged to remove urea. The proteins were then alkylated by iodoacetamide at room temperature for $20 \mathrm{~min}$ (in the dark) and digested with sequence-grade modified trypsin (Promega) and lyophilized. 


\section{LCMS/MS analysis}

Solvent A $(0.1 \%$ formic acid, $30 \mu \mathrm{L})$ was used for resuspending peptides. Separations were performed with an EASY-nano-LC 1200 system (Thermo Fisher Scientific). Peptide sample $(6 \mu \mathrm{L})$ was loaded into a trap column (C18, $75 \mu \mathrm{m} \times 2$ $\mathrm{cm}$, flow rate: $300 \mathrm{~nL} / \mathrm{min}$ ), and subsequently separated and loaded onto an analytical column $(\mathrm{C} 18,75 \mu \mathrm{m} \times 50 \mathrm{~cm})$ using a linear gradient of 5 $-38 \%$ B $(0.1 \%$ formic acid in ACN) for $120 \mathrm{~min}$. A $2 \mathrm{kV}$ electrospray voltage between the sprayer and ion inlet of the mass spectrometer was utilized in the study.

\section{Identification of DEPs}

PEAKS Studio (version 8.5, Bioinformatics Solutions Inc., Waterloo, Canada) was used to analyze tandem mass spectra. PEAKS DB was used to search the UniProt-mouse database (ver.201711, 52194 entries). The search parameters were 0.05 and $7 \mathrm{ppm}$ for the fragment and parent ions mass tolerances, respectively. The fixed modification was carbamidomethylation $(\mathrm{C})$, while the variable modifications were deamidation (NQ), oxidation $(\mathrm{M})$, and acetylation (Protein N-term). Peptides were filtered with $1 \%$ FDR and 1 unique. The abundance of peptide and protein was calculated using ANOVA. The averaging the abundance of all peptides was normalization using medians. Protein with fold-changes over 1.5 and at least 2 unique peptides with significance over 13 ( $p<$ $0.05)$ was considered to be a Differentlyexpressed protein (DEP).

\section{Bioinformatics analysis}

The obtained DEPs were analyzed using three databases: Kyoto Encyclopedia of Genes and Genomes (KEGG), Gene Ontology (GO), and the Clusters of Orthologous Groups (KOGs) databases. The interaction network of DEPs was built with the STRING.

\section{Western blot analysis}

Uterus tissue from each mouse was used to

Table 1: Effect of NZD on writhing reaction, analgesia and serum PGE2 and PGF2 $\alpha$ levels of PD model mice

\begin{tabular}{lllll}
\hline Group & $\begin{array}{l}\text { Number of writhes in 30 } \\
\text { min }\end{array}$ & Analgesia (\%) & PGE2 $(\mathbf{p g} / \mathbf{m L})$ & $\begin{array}{l}\text { PGF2 } \alpha \\
(\mathbf{p g} / \mathbf{m L})\end{array}$ \\
\hline Control & $0.00 \pm 0.00$ & 100.00 & $70.60 \pm 19.31$ & $239.46 \pm 27.41$ \\
Model & $61.30 \pm 9.56$ & 0.00 & $142.80 \pm 12.79^{\cdots}$ & $718.27 \pm 35.07$ \\
Treatment & $23.40 \pm 13.60^{\# \#}$ & 61.82 & $35.40 \pm 15.01^{\# \#}$ & $317.87 \pm 72.28^{\# \#}$ \\
\hline
\end{tabular}

${ }^{* *} p<0.01$, compared to control group; ${ }^{\# \#} p<0.01$, compared to model group $(n=10)$. extract protein using RIPA lysis buffer containing $1 \%$ PMSF and cocktail (Beyotime, Haimen, China). BCA assay was employed to estimate the protein concentration. The protein was separated by $8-10 \%$ SDS-PAGE and transferred to polyvinylidene difluoride (PVDF) membranes. After blocking with non-fat milk (5\%), the membranes were then incubated overnight with anti-vinculin, anti-caveolin, anti-stat1, anti-rock1 and anti-GAPDH at $4{ }^{\circ} \mathrm{C}$. The membranes were washed thrice with TBST buffer and then incubated with secondary antibodies at room temperature for $1 \mathrm{~h}$. Enhanced chemiluminescence (ECL) reagent (Thermo Fisher Scientific, Inc.) was used for the detection of interest protein bands. Image J v1.48u software (National Institutes of Health, Bethesda, MD, USA) was employed to analyze the relative optical densities of interest bands.

\section{Statistical analysis}

Statistical analysis was performed with SPSS 19.0 software (IBM Corp., NY). All data are expressed as mean \pm standard deviation (SD). The differences between two groups were analyzed by Student's $t$-test. One-way ANOVA was used for multiple-group comparisons. Statistical significance was assumed at $p<0.05$.

\section{RESULTS}

\section{Effect of NZD on writhing in primary dysmenorrhea}

As shown in Table 1, compared with the control mice, a remarkable increased number of writhes was observed in model mice, indicating the successful establishment of PD model. Treatment with NZD significantly reduced the number of writhes $(p<0.01)$, and the percentage analgesia was 61.82. Model mice showed significant increases in the levels of serum PGE2 and PGF2 $\alpha$. However, administration of NZD induced a remarkable decrease in the levels PGE2 and PGF2 $\alpha(p<0.01)$. These findings suggest that NZD can significantly relieved PD. 
Identification of DEPs in uterus of PD mice administered NZD

Nano-HPLC-MS/MS was applied to identify DEPs in uterine tissues in the three groups. As shown in Table 2, a total of 556 DEPs were identified between control group and model group, out of which 245 were up-regulated, while 311 were down-regulated. Four hundred and four (404) DEPs were identified between the model group and treatment group, 238 of which were up-regulated, while 166 were down-regulated. There were 471 DEPs between the control group and the treatment group, with 267 up-regulated and 204 down-regulated. The DEPs among the three groups were then further analyzed via identification of the up-regulated or downregulated proteins in model and treatment groups. Sixty-six proteins were up-regulated in model group and down-regulated in treatment group, while 38 proteins which were downregulated in model group were up-regulated in treatment group. These results are displayed in Table 3.

\section{GO analysis}

To extract information relevant to involved pathways of DEPs, the protein data obtained were analyzed using DAVID network analysis tool. Moreover, GO analysis was carried out on cellular components (CC), molecular functions (MF), and biological processes (BPs) associated with the DEPs. In the BP analysis, majority of DEPs were associated with immune response, immune system process, regulation of localization and single-organism transport (Figure 1). The CC analysis showed that most of DEPs were present in the cytoplasm (Figure 1). Molecular functional classification of DEPs showed that DEPs were mainly involved in protein binding, catalytic activity and hydrolase activity (Figure 1).

\section{KEGG pathway analysis}

The results of KEGG analysis revealed that the DEPs were significantly associated with tuberculosis, Staphylococcus aureus infection, leukocyte transendothelial migration and phagosome. The results also indicated that the DEPs were associated with Parkinson's disease, Fc gamma R-mediated phagocytosis and chemokine signaling pathway (Figure 2).

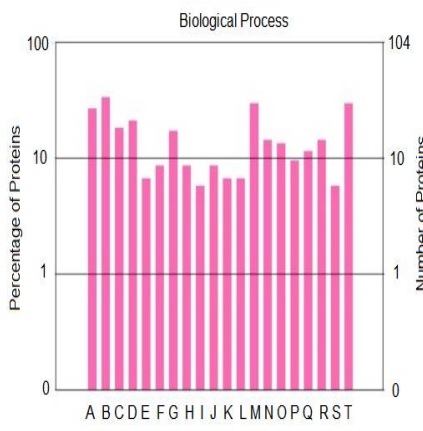

A immune response

B. immune system process immune system process regulation of immune system process F. blood coaguation G. boositive regulation of transport H. coagulation 1. regulation myeloid leukocyte mediated immunity J. hemostasis K. myeloid leukocyte mediated immunity L. platelet activation

M. regulation of localization N. immune effector process . positive regulation of immune respo
P. regulation of body fluid levels Q. segulation of body fluid levels R. regulation of immune response

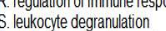
T. single-organism transport

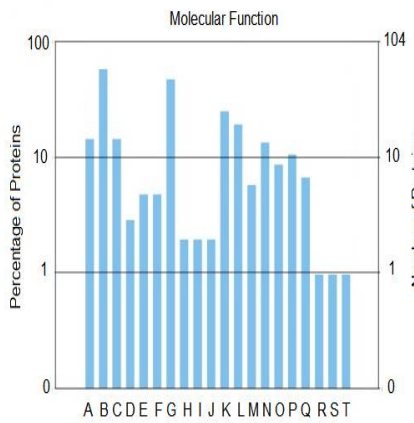

A. protein complex binding B. protein binding C. macromolecular complex binding D. immunoglobulin binding E. cysteine type endopepitidase activity F. oxidoreductase activity on NADPH

G. catalytic activity'
H. immunoglobulin receptor activity 1. IgG binding tor binding K. hydrolase activity M. cysteine trpe peptidase activit N. cytoskeletal protein binding Q. cell achesion molecular binding P. oxidoreductase activity Q. ubiquitin-lke protein Igase binding R. glycine amidinotransterase acti S. amidinotransierase activity

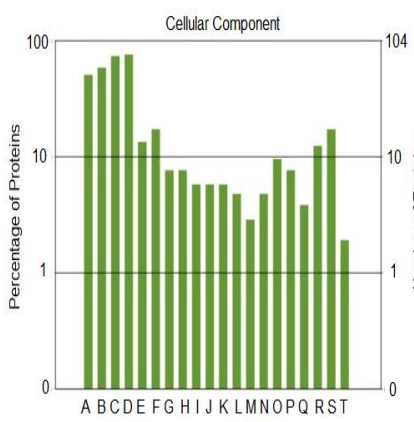

A cytoplasmic part A. cytoplasmic
B. cytoplasm D. intracelluar E. membrane protein complex F. extracelluar region part $G$. adherens junction H. anchoring junction - local adnesion $\mathrm{K}$. cell-substrate junction L. integin complex M. podosome

ex involved in cell adhesion

Q. actin cytoskeleton Q. oxidoreduclase complex R. extracelular space S. extracelluar region T. fascia adherens

Figure 1: GO annotation of DEPs in biological process (BP), cellular component (CC) and molecular function (MF)

Table 2: Number of DEP in uteruses of control, model and treatment groups

\begin{tabular}{llll}
\hline Group & $\begin{array}{l}\text { Number of } \\
\text { DEPs }\end{array}$ & $\begin{array}{l}\text { Number of up- } \\
\text { regulated } \\
\text { proteins }\end{array}$ & $\begin{array}{l}\text { Number of down- } \\
\text { regulated } \\
\text { proteins }\end{array}$ \\
\hline Control group vs model group & 556 & 245 & 311 \\
Model group vs treatment group & 404 & 238 & 166 \\
Control group vs treatment group & 471 & 267 & 204 \\
\hline
\end{tabular}


Table 3: Comparison of differentially-expressed proteins (DEPs) in uterus among control, model and treatment groups

\begin{tabular}{|c|c|c|c|c|c|c|c|c|}
\hline \multirow{2}{*}{$\begin{array}{l}\text { Uniprot } \\
\text { accession no. }\end{array}$} & \multirow[t]{2}{*}{ Protein description } & \multirow{2}{*}{$\begin{array}{l}\text { Gene } \\
\text { symbol }\end{array}$} & \multicolumn{2}{|c|}{ Fold change ratio } & \multicolumn{2}{|c|}{$\boldsymbol{P}$} & \multicolumn{2}{|c|}{ Tendency } \\
\hline & & & A & $\mathrm{B}$ & A & $\mathrm{B}$ & $\mathrm{A}$ & $\mathrm{B}$ \\
\hline E9QPE7 & Myosin-11 & Myh11 & 0.437 & 1.563 & 0.038 & 0.048 & Do & Up \\
\hline P31001 & Desmin & Des & 0.488 & 1.845 & 0.011 & 0.008 & Do & Up \\
\hline E9PZ16 & $\begin{array}{l}\text { Basement membrane-specific heparan sulfate } \\
\text { proteoglvcan core protein }\end{array}$ & Hspg2 & 0.397 & 1.652 & 0.029 & 0.037 & Do & Up \\
\hline Q80X90 & Filamin-B & FInb & 1.813 & 0.633 & 0.009 & 0.008 & Up & Do \\
\hline Q9JJZ2 & Tubulin alpha-8 chain & Tuba8 & 1.614 & 0.382 & 0.031 & 0.048 & Up & Do \\
\hline Q64727 & Vinculin & $\mathrm{Vcl}$ & 0.663 & 1.755 & 0.037 & 0.042 & Do & Up \\
\hline E9PV24 & Fibrinogen alpha chain & Fga & 1.912 & 0.483 & 0.011 & 0.019 & Up & Do \\
\hline Q61233 & Plastin-2 & Lcp1 & 2.013 & 0.423 & 0.006 & 0.008 & Up & Do \\
\hline P00920 & Carbonic anhydrase 2 & $\mathrm{Ca} 2$ & 1.914 & 0.659 & 0.033 & 0.045 & Up & Do \\
\hline P51661 & $\begin{array}{l}\text { Corticosteroid 11-beta-dehydrogenase isozyme } \\
2\end{array}$ & Hsd11b2 & 0.421 & 1.606 & 0.036 & 0.048 & Do & Up \\
\hline P97449 & Aminopeptidase $\mathrm{N}$ & Anpep & 0.388 & 1.563 & 0.015 & 0.018 & Do & Up \\
\hline P01029 & Complement C4-B & $\mathrm{C} 4 \mathrm{~b}$ & 1.214 & 0.437 & 0.031 & 0.040 & Up & Do \\
\hline A0A0R4J1B4 & Integrin alpha-M & Itgam & 2.131 & 0.590 & 0.001 & 0.004 & Up & Do \\
\hline Q9D379 & Epoxide hydrolase 1 & Ephx1 & 0.294 & 1.970 & 0.006 & 0.018 & Do & Up \\
\hline P25688 & Uricase & Uox & 2.114 & 0.576 & 0.032 & 0.041 & Up & Do \\
\hline Q8C3V4 & Signal transducer and activator of transcription & Stat1 & 1.985 & 0.438 & 0.003 & 0.002 & Up & Do \\
\hline P16125 & L-lactate dehydrogenase $\mathrm{B}$ chain & Ldhb & 0.214 & 1.597 & 0.002 & 0.003 & Do & Up \\
\hline P14873 & Microtubule-associated protein 1B & Map1b & 0.564 & 1.811 & 0.041 & 0.048 & Do & Up \\
\hline P01865 & Ig gamma-2A chain $\mathrm{C}$ region & Igh-1a & 1.354 & 0.623 & 0.039 & 0.045 & Up & Do \\
\hline P30681 & High mobility group protein B2 & Hmgb2 & 1.974 & 0.647 & 0.019 & 0.026 & Up & Do \\
\hline Q61599 & Rho GDP-dissociation inhibitor 2 & Arhgdib & 2.231 & 0.459 & 0.015 & 0.010 & Up & Do \\
\hline Q54218 & Integrin beta & Itgb2 & 2.223 & 0.545 & 0.009 & 0.010 & Up & Do \\
\hline P68037 & Ubiquitin-conjugating enzyme E2 L3 & Ube2I3 & 1.991 & 0.623 & 0.025 & 0.038 & Up & Do \\
\hline Q05144 & Ras-related C3 botulinum toxin substrate 2 & Rac2 & 2.412 & 0.447 & 0.001 & 0.004 & Up & Do \\
\hline Q9CZS1 & Aldehyde dehydrogenase X mitochondrial & Aldh1b1 & 0.477 & 4.196 & 0.026 & 0.010 & Do & Up \\
\hline Q8K1B8 & Fermitin family homolog 3 & Fermt3 & 1.876 & 0.484 & 0.031 & 0.021 & Up & Do \\
\hline
\end{tabular}


Table 3: Comparison of differentially-expressed proteins (DEPs) in uterus among control, model and treatment groups

\begin{tabular}{|c|c|c|c|c|c|c|c|c|}
\hline \multirow{2}{*}{$\begin{array}{l}\text { Uniprot } \\
\text { accession no. }\end{array}$} & \multirow[t]{2}{*}{ Protein description } & \multirow{2}{*}{$\begin{array}{l}\text { Gene } \\
\text { symbol }\end{array}$} & \multicolumn{2}{|c|}{ Fold change ratio } & \multicolumn{2}{|c|}{$\boldsymbol{P}$} & \multicolumn{2}{|c|}{ Tendency } \\
\hline & & & A & $\mathrm{B}$ & A & $\mathrm{B}$ & $\mathrm{A}$ & $\mathrm{B}$ \\
\hline Q9ESB3 & Histidine-rich glycoprotein & $\mathrm{Hrg}$ & 2.223 & 0.549 & 0.008 & 0.011 & Up & Do \\
\hline AOA171EBL2 & E3 ubiquitin-protein ligase RNF213 & Rnf213 & 1.968 & 0.362 & 0.012 & 0.014 & Up & Do \\
\hline P25911 & Tyrosine-protein kinase Lyn & Lyn & 1.963 & 0.597 & 0.015 & 0.017 & Up & Do \\
\hline P43275 & Histone H1.1 & Hist1h1a & 2.218 & 0.576 & 0.007 & 0.010 & Up & Do \\
\hline P49817 & Caveolin-1 & Cav1 & 0.254 & 2.009 & 0.008 & 0.006 & Do & Up \\
\hline P35385 & Heat shock protein beta- 7 & Hspb7 & 0.873 & 2.011 & 0.038 & 0.045 & Do & Up \\
\hline S4R1M0 & Receptor-type tyrosine-protein phosphatase C & Ptprc & 1.752 & 0.414 & 0.024 & 0.030 & Up & Do \\
\hline Q61093 & Cytochrome b-245 heavy chain 1 & Cybb & 2.014 & 0.536 & 0.001 & 0.002 & Up & Do \\
\hline e & $\begin{array}{l}\text { Basic leucine zipper and W2 domain-containing } \\
\text { protein } 2\end{array}$ & Bzw2 & 0.374 & 1.598 & 0.001 & 0.002 & Do & Up \\
\hline P97821 & Dipeptidyl peptidase 1 & Ctsc & 1.797 & 0.495 & 0.012 & 0.016 & Up & Do \\
\hline e & Long-chain-fatty-acid--CoA ligase 3 & Acsl3 & 1.895 & 0.585 & 0.011 & 0.010 & Up & Do \\
\hline e & $\begin{array}{l}\text { NADH dehydrogenase [ubiquinone] } 1 \text { alpha } \\
\text { subcomplex subunit } 10 \text { mitochondrial }\end{array}$ & Ndufa10 & 0.373 & 1.623 & 0.003 & 0.005 & Do & Up \\
\hline Q9Z0P5 & Twinfilin-2 & Twf2 & 1.987 & 0.639 & 0.019 & 0.024 & Up & Do \\
\hline Q8K1J6 & CCA tRNA nucleotidyltransferase 1 & Trnt1 & 2.013 & 0.487 & 0.017 & 0.022 & Up & Do \\
\hline Q60770 & Syntaxin-binding protein 3 & Stxbp3 & 0.434 & 1.601 & 0.029 & 0.034 & Do & Up \\
\hline Q7TMF3 & $\begin{array}{l}\text { NADH dehydrogenase [ubiquinone] } 1 \text { alpha } \\
\text { subcomplex subunit } 12\end{array}$ & Ndufa12 & 0.383 & 1.552 & 0.025 & 0.038 & Do & Up \\
\hline G3UW94 & MCG14259 isoform CRA_b & U2af1 & 1.646 & 0.473 & 0.034 & 0.045 & Up & Do \\
\hline P56695 & Wolframin & Wfs 1 & 0.593 & 1.668 & 0.019 & 0.035 & Do & Up \\
\hline Q8K124 & $\begin{array}{l}\text { Pleckstrin homology domain-containing family } \mathrm{O} \\
\text { member } 2\end{array}$ & Plekho2 & 2.015 & 0.475 & 0.002 & 0.004 & Up & Do \\
\hline O35744 & Chitinase-like protein 3 & Chil3 & 1.030 & 0.199 & 0.041 & 0.047 & Up & Do \\
\hline P49710 & Hematopoietic lineage cell-specific protein & Hcls1 & 1.717 & 0.428 & 0.009 & 0.011 & Up & Do \\
\hline Q80WQ2 & Protein VAC14 homolog & Vac14 & 0.252 & 1.644 & 0.011 & 0.024 & Do & Up \\
\hline Q9D964 & Glycine amidinotransferase mitochondrial & Gatm & 2.556 & 0.494 & 0.001 & 0.000 & Up & Do \\
\hline 009117 & Synaptophysin-like protein 1 & Sypl1 & 0.686 & 2.134 & 0.024 & 0.039 & Do & Up \\
\hline O89017 & Legumain & Lgmn & 1.881 & 0.5900 & 0.018 & 0.010 & Up & Do \\
\hline P97426 & Eosinophil cationic protein 1 & Ear1 & 1.667 & 0.598 & 0.027 & 0.038 & Up & Do \\
\hline AOA0B4J1G1 & Fc receptor IgG low affinity IIb & Fcgr2b & 1.737 & 0.286 & 0.008 & 0.006 & Up & Do \\
\hline e & Large proline-rich protein BAG6 & Bag6 & 1.223 & 0.619 & 0.041 & 0.037 & Up & Do \\
\hline Q8BLY1 & SPARC-related modular calcium-binding protein & Smoc1 & 0.667 & 2.390 & 0.039 & 0.049 & Do & Up \\
\hline
\end{tabular}


Table 3: Comparison of differentially-expressed proteins (DEPs) in uterus among control, model and treatment groups

\begin{tabular}{|c|c|c|c|c|c|c|c|c|}
\hline \multirow{2}{*}{$\begin{array}{l}\text { Uniprot } \\
\text { accession no. }\end{array}$} & \multirow[t]{2}{*}{ Protein description } & \multirow{2}{*}{$\begin{array}{l}\text { Gene } \\
\text { symbol }\end{array}$} & \multicolumn{2}{|c|}{ Fold change ratio } & \multicolumn{2}{|c|}{$\boldsymbol{P}$} & \multicolumn{2}{|c|}{ Tendency } \\
\hline & & & $\mathrm{A}$ & $\mathrm{B}$ & A & $\mathrm{B}$ & $\mathrm{A}$ & $\mathrm{B}$ \\
\hline P70335 & Rho-associated protein kinase 1 & Rock1 & 1.414 & 0.377 & 0.018 & 0.027 & Up & Do \\
\hline Q9D1J3 & SAP domain-containing ribonucleoprotein & Sarnp & 1.919 & 0.654 & 0.018 & 0.022 & Up & Do \\
\hline Q8BYA0 & Tubulin-specific chaperone D & Tbcd & 0.626 & 2.160 & 0.028 & 0.005 & Do & Up \\
\hline Q07456 & Protein AMBP & Ambp & 1.558 & 0.534 & 0.029 & 0.033 & Up & Do \\
\hline G5E814 & MCG5603 & Ndufa11 & 1.663 & 0.578 & 0.021 & 0.018 & Up & Do \\
\hline 035601 & FYN-binding protein 1 & Fyb1 & 1.364 & 0.580 & 0.037 & 0.049 & Up & Do \\
\hline 070370 & Cathepsin S & Ctss & 1.414 & 0.428 & 0.031 & 0.029 & Up & Do \\
\hline Q3UIR3 & E3 ubiquitin-protein ligase DTX3L & $\mathrm{Dtx} 3 \mathrm{I}$ & 1.818 & 0.643 & 0.022 & 0.036 & Up & Do \\
\hline Q60648 & Ganglioside GM2 activator & $\mathrm{Gm} 2 \mathrm{a}$ & 0.418 & 2.159 & 0.026 & 0.043 & Do & Up \\
\hline Q6P9Q6 & FK506-binding protein 15 & Fkbp15 & 2.073 & 0.617 & 0.011 & 0.019 & Up & Do \\
\hline Q8BWZ3 & $\begin{array}{l}\text { N-alpha-acetyltransferase } 25 \text { NatB auxiliary } \\
\text { subunit }\end{array}$ & Naa25 & 0.593 & 1.827 & 0.007 & 0.011 & Do & Up \\
\hline O89110 & Caspase-8 & Casp8 & 2.141 & 0.559 & 0.002 & 0.005 & Up & Do \\
\hline Q78J03 & Methionine-R-sulfoxide reductase B2 & Msrb2 & 0.492 & 1.972 & 0.023 & 0.037 & Do & Up \\
\hline P31725 & Protein S100-A9 & S100a9 & 2.015 & 0.378 & 0.009 & 0.014 & Up & Do \\
\hline A2APF7 & Z-DNA-binding protein 1 & Zbp1 & 2.221 & 0.306 & 0.001 & 0.007 & Up & Do \\
\hline Q91Z40 & Gbp6 protein & Gbp7 & 1.823 & 0.324 & 0.021 & 0.018 & Up & Do \\
\hline Q9JHK5 & Pleckstrin & Plek & 1.973 & 0.552 & 0.010 & 0.017 & Up & Do \\
\hline Q9R0P9 & $\begin{array}{l}\text { Ubiquitin carboxyl-terminal hydrolase isozyme } \\
\text { L1 }\end{array}$ & Uchl1 & 0.545 & 2.039 & 0.013 & 0.027 & Do & Up \\
\hline Q8BPU7 & Engulfment and cell motility protein 1 & Elmo1 & 1.662 & 0.357 & 0.022 & 0.043 & Up & Do \\
\hline A2AP32 & $\begin{array}{l}\text { NADH dehydrogenase [ubiquinone] } 1 \text { beta } \\
\text { subcomplex subunit } 6\end{array}$ & Ndufb6 & 0.587 & 4.531 & 0.035 & 0.008 & Do & Up \\
\hline Q3ULD5 & Methylcrotonoyl-CoA carboxylase beta chain & Mccc2 & 0.212 & 1.703 & 0.003 & 0.006 & Do & Up \\
\hline 070200 & Allograft inflammatory factor 1 & Aif1 & 2.313 & 0.509 & 0.002 & 0.010 & Up & Do \\
\hline Q9QXD8 & LIM domain-containing protein 1 & Limd1 & 2.432 & 0.657 & 0.000 & 0.004 & Up & Do \\
\hline Q8C3J5 & Dedicator of cytokinesis protein 2 & Dock2 & 1.373 & 0.149 & 0.011 & 0.040 & Up & Do \\
\hline Q99LI7 & Cleavage stimulation factor subunit 3 & Cstf3 & 0.245 & 1.940 & 0.032 & 0.028 & Do & Up \\
\hline Q3UVK0 & Endoplasmic reticulum metallopeptidase 1 & Ermp1 & 1.562 & 0.665 & 0.014 & 0.017 & Up & Do \\
\hline Q02105 & Complement $\mathrm{C} 1 \mathrm{q}$ subcomponent subunit $\mathrm{C}$ & $\mathrm{C} 1 \mathrm{qc}$ & 1.636 & 0.485 & 0.019 & 0.028 & Up & Do \\
\hline P57787 & Monocarboxylate transporter 4 & Slc16a3 & 1.921 & 0.511 & 0.021 & 0.013 & Up & Do \\
\hline Q9R1J0 & $\begin{array}{l}\text { Sterol-4-alpha-carboxylate 3-dehydrogenase } \\
\text { decarboxylating }\end{array}$ & Nsdhl & 2.015 & 0.636 & 0.014 & 0.026 & Up & Do \\
\hline Q64282 & $\begin{array}{l}\text { Interferon-induced protein with tetratricopeptide } \\
\text { repeats } 1\end{array}$ & Ifit1 & 1.999 & 0.262 & 0.004 & 0.008 & Up & Do \\
\hline
\end{tabular}


Table 3: Comparison of differentially-expressed proteins (DEPs) in uterus among control, model and treatment groups

\begin{tabular}{|c|c|c|c|c|c|c|c|c|}
\hline \multirow{2}{*}{$\begin{array}{l}\text { Uniprot } \\
\text { accession no. }\end{array}$} & \multirow[t]{2}{*}{ Protein description } & \multirow{2}{*}{$\begin{array}{l}\text { Gene } \\
\text { symbol }\end{array}$} & \multicolumn{2}{|c|}{ Fold change ratio } & \multicolumn{2}{|l|}{$P$} & \multicolumn{2}{|c|}{ Tendency } \\
\hline & & & $\mathrm{A}$ & $\mathrm{B}$ & A & $\mathrm{B}$ & A & $\mathrm{B}$ \\
\hline Q9DB73 & \multirow{4}{*}{$\begin{array}{l}\text { NADH-cytochrome b5 reductase } 1 \\
\text { Interferon-induced double-stranded RNA- } \\
\text { activated protein kinase } \\
\text { MICOS complex subunit Mic } 25 \\
\text { High affinity immunoglobulin epsilon receptor } \\
\text { subunit gamma }\end{array}$} & Cyb5r1 & 0.445 & 2.416 & 0.010 & 0.025 & Do & Up \\
\hline Q03963 & & Eif2ak2 & 1.844 & 0.546 & 0.027 & 0.039 & Up & Do \\
\hline Q91VN4 & & Chchd6 & 0.461 & 1.724 & 0.013 & 0.025 & Do & Up \\
\hline P20491 & & Fcer1g & 1.525 & 0.484 & 0.023 & 0.015 & Up & Do \\
\hline $\begin{array}{l}\text { Q8BUK6 } \\
\text { Q62084 } \\
\text { Q9EQ32 } \\
\text { Q8K1R3 } \\
\text { Q91Z50 }\end{array}$ & $\begin{array}{l}\text { Protein Hook homolog } 3 \\
\text { Protein phosphatase } 1 \text { regulatory subunit 14B } \\
\text { Phosphoinositide } 3 \text {-kinase adapter protein } 1 \\
\text { Polyribonucleotide nucleotidyltransferase } 1 \\
\text { Flap endonuclease } 1\end{array}$ & $\begin{array}{l}\text { Hook3 } \\
\text { Ppp1r14b } \\
\text { Pik3ap1 } \\
\text { Pnpt1 } \\
\text { Fen1 }\end{array}$ & $\begin{array}{l}0.915 \\
0.321 \\
2.156 \\
0.858 \\
1.752\end{array}$ & $\begin{array}{l}2.178 \\
1.717 \\
0.243 \\
7.289 \\
0.411\end{array}$ & $\begin{array}{l}0.038 \\
0.034 \\
0.000 \\
0.001 \\
0.016\end{array}$ & $\begin{array}{l}0.046 \\
0.029 \\
0.002 \\
0.004 \\
0.026\end{array}$ & $\begin{array}{l}\text { Do } \\
\text { Do } \\
\text { Up } \\
\text { Do } \\
\text { Up }\end{array}$ & $\begin{array}{l}\text { Up } \\
\text { Up } \\
\text { Do } \\
\text { Up } \\
\text { Do }\end{array}$ \\
\hline e & $\begin{array}{l}\text { FYVE and coiled-coil domain-containing protein } \\
1\end{array}$ & Fyco1 & 1.842 & 0.354 & 0.037 & 0.024 & Up & Do \\
\hline $\begin{array}{l}\text { Q9Z1Q2 } \\
\text { Q5SWD9 } \\
\text { Q8C0Z1 } \\
\text { P13597 } \\
\text { e } \\
\text { Q9R099 }\end{array}$ & $\begin{array}{l}\text { Protein ABHD16A } \\
\text { Pre-rRNA-processing protein TSR1 homolog } \\
\text { Protein FAM234A } \\
\text { Intercellular adhesion molecule } 1 \\
\text { Protein THEMIS2 } \\
\text { Transducin beta-like protein } 2\end{array}$ & $\begin{array}{l}\text { Abhd16a } \\
\text { Tsr1 } \\
\text { Fam234 } \\
\text { Icam1 } \\
\text { Themis2 } \\
\text { Tbl2 }\end{array}$ & $\begin{array}{l}0.553 \\
0.663 \\
0.515 \\
1.641 \\
1.717 \\
0.373\end{array}$ & $\begin{array}{l}1.541 \\
1.917 \\
2.496 \\
0.218 \\
0.431 \\
2.090\end{array}$ & $\begin{array}{l}0.021 \\
0.041 \\
0.009 \\
0.031 \\
0.019 \\
0.011\end{array}$ & $\begin{array}{l}0.012 \\
0.040 \\
0.002 \\
0.029 \\
0.025 \\
0.019\end{array}$ & $\begin{array}{l}\text { Do } \\
\text { Do } \\
\text { Do } \\
\text { Up } \\
\text { Up } \\
\text { Do }\end{array}$ & $\begin{array}{l}\text { Up } \\
\text { Up } \\
\text { Up } \\
\text { Do } \\
\text { Do } \\
\text { Up }\end{array}$ \\
\hline P26151 & $\begin{array}{l}\text { High affinity immunoglobulin gamma Fc } \\
\text { receptor I }\end{array}$ & Fcgr1 & 1.565 & 0.290 & 0.018 & 0.029 & Up & Do \\
\hline
\end{tabular}

A: comparison of control group and model group; B: comparison of model group and treatment group. Up = up-regulation; Do = down-regulation 


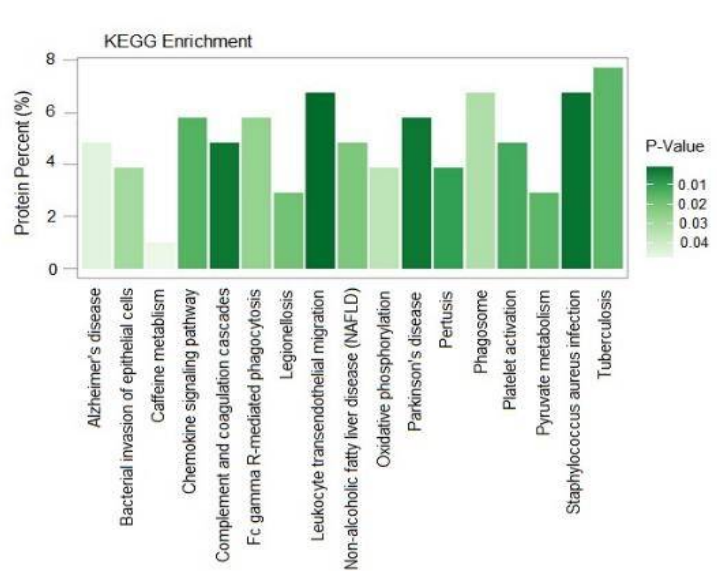

Figure 2: Distribution of enriched KEGG pathway. Columns represents related pathways. Midnight green represents smaller $p$ values) and lighter green represents bigger $p$ values

\section{KOGs analysis}

The results of KOGs analysis showed that the functions of the DEPs were mostly in information storage and processing, cellular processes and signaling, and metabolism (Figure 3 ). The results indicated that these processes may be involved in the therapeutic effect of NZD.

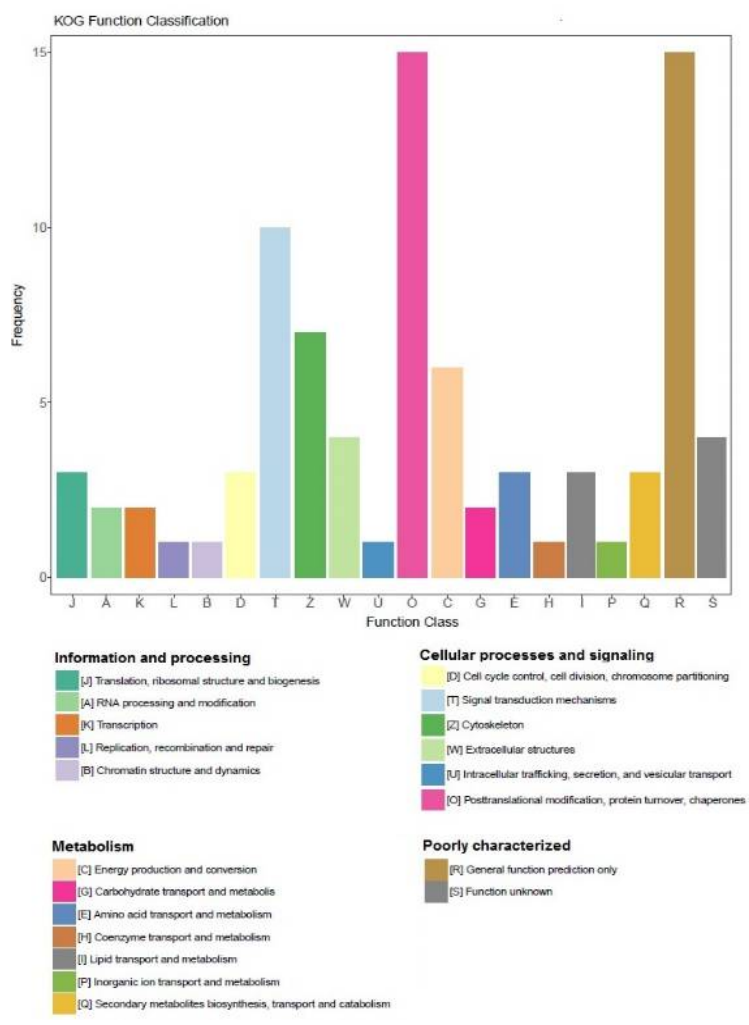

Figure 3: KOGs analysis of DEPs. The different colored columns refer to different functions. The frequency of proteins enriched in the function refer to the value of the ordinate.

\section{PPI analysis}

For further exploring the mechanisms involved in the protective effect of NZD, STRING database was used to construct the PPI network of the DEPs. As shown in Figures 4, a total of 53 DEPs in the map have direct or indirect links.

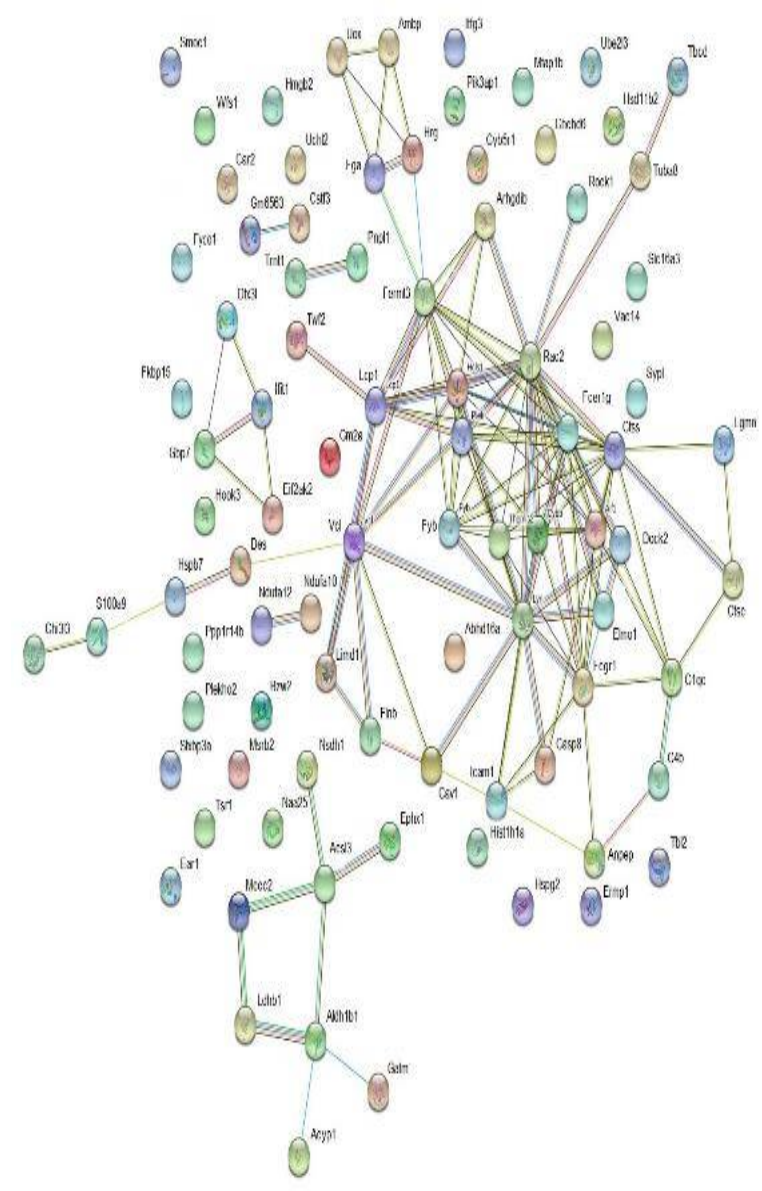

Figure 4: Interaction networks of DEPs. Protein association network of the DEPs were constructed after searching STRING database with a confidence cutoff of 0.6. Proteins are indicated by nodes. Thickness of lines represents the confidence level (0.6 $-0.9)$.

\section{Validation by western blot on DEPs}

As shown in Fig.5, the expression levels of Stat1 and Rock1 were significantly elevated in model group and down-regulated in treatment group, while vinculin and caveolin-1 showed significant decreases in model group and increases in treatment group. These results were consistent with the observations in proteomics analysis.

\section{DISCUSSION}

Although NZD has been used in China to treat PD in clinics for many years, the underlying 
mechanism remains largely unknown. To the best of the authors' knowledge, the present study is the first to use label-free proteomic based method to investigate the mechanism of NZD on a PD model. The results showed that NZD reduced oxytocin-induced writhing response after oxytocin injection in PD mice. The serum levels of PGE2 and PGF2 $\alpha$, which are regarded as the most critical pain factors in PD [17], were significantly decreased after NZD administration. These findings suggest that NZD exerts a significant analgesic effect in PD mice.

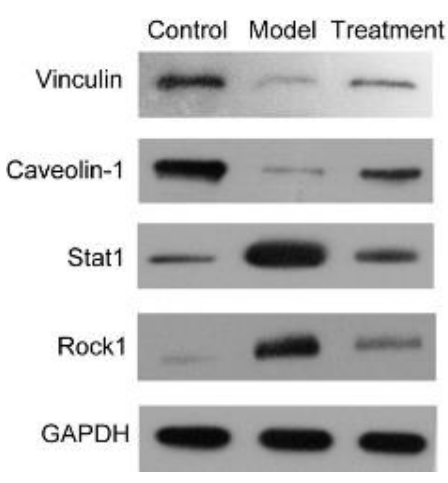

Figure 5: Western blot analysis confirmed DEPS initially identified using quantitative proteomics method. The expressions of vinculin, caveolin-1, Stat1 and Rock1 in uteruses of mice in the three groups were analyzed using western blot, with GAPDH as the loading control

Label-free quantitative proteomics is useful in searching for disease-associated factors and has been used to investigate the mechanism of TCM in recent years [11-14]. The present study identified 38 up-regulated DEPs and 66 downregulated DEPs after NZD treatment. The GO and KEGG analyses revealed significant alteration of functions and signaling pathways in PD mice after NZD administration. These changes affected protein binding, immune response, catalytic activity and chemokine signaling pathway. These GOs and pathways may play important roles in the analgesic action of NZD. The results of KOGs analysis revealed that the functions of the DEPs mainly involved in metabolism, cellular processes and signaling, information storage and processing.

The accuracy of proteomics was verified using western blotting with respect to the expressions of vinculin, caveolin-1, Stat1 and Rock1 in the three groups. The levels of Stat1 and Rock1 in uteruses were elevated in model group and down-regulated in treatment group. Vinculin and caveolin-1 showed decreases in model group and increases in treatment group. These results confirmed the credibility of proteomic analysis.

\section{CONCLUSION}

The proteomic studies in the present investigation have revealed a number of DEPs involved in the response to NZD administration in PD mice. It is hoped that these findings will provide a database resource for further investigations on the mechanisms involved in the protective effect of NZD against PD.

\section{DECLARATIONS}

\section{Acknowledgement}

This work was supported by the Project of Taicang Science and Technology (no. TC2017YYJC03).

\section{Conflict of interest}

No conflict of interest is associated with this work

\section{Contribution of authors}

We declare that this work was done by the authors named in this article, and all liabilities pertaining to claims relating to the content of this article will be borne by the authors. Qi-Bin Lu designed all the experiments and revised the manuscript. Ya-Zhen Xie and Jian-Qiang Qian performed the experiments, while Ya-Zhen Xie wrote the manuscript.

\section{Open Access}

This is an Open Access article that uses a funding model which does not charge readers or their institutions for access and distributed under the terms of the Creative Commons Attribution License (http://creativecommons.org/licenses/by/ 4.0) and the Budapest Open Access Initiative (http://www.budapestopenaccessinitiative.org/rea d), which permit unrestricted use, distribution, and reproduction in any medium, provided the original work is properly credited.

\section{REFERENCES}

1. Rencz F, Pentek M, Stalmeier PFM, Brodszky V, Ruzsa G, Gradvohl E, Baji P, Gulacsi L. Bleeding out the quality-adjusted life years: evaluating the burden of primary dysmenorrhea using time trade-off and willingness-to-pay methods. Pain 2017; 158(11): 22592267.

2. Suvitie P. Dysmenorrhea in teenagers. Duodecim 2017; 133(3): 285-291.

3. Payne LA, Rapkin AJ, Seidman LC, Zeltzer LK, Tsao JC. Experimental and procedural pain responses in primary 
dysmenorrhea: a systematic review. J Pain Res 2017; 10: 2233-2246.

4. Bernardi M, Lazzeri L, Perelli F, Reis FM, Petraglia F. Dysmenorrhea and related disorders. F1000Res 2017; 6: 1645.

5. Davis AR, Westhoff $C$, O'Connell $K$, Gallagher N. Oral contraceptives for dysmenorrhea in adolescent girls: a randomized trial. Obstet Gynecol 2005; 106(1): 97-104.

6. Nazar $H$, Usmanghani $K$. Clinical evaluation to assess the safety and efficacy of coded herbal medicine "Dysmo-off" versus allopathic medicine "Diclofenac sodium" for the treatment of primary dysmenorrhea. $J$ Herb Pharmacother 2006; 6(1): 21-39.

7. Oya A, Oikawa $T$, Nakai A, Takeshita $T$, Hanawa $T$. Clinical efficacy of Kampo medicine (Japanese traditional herbal medicine) in the treatment of primary dysmenorrhea. J Obstet Gynaecol Res 2008; 34(5): 898-908.

8. Gao L, Jia C, Zhang H, Ma C. Wenjing decoction (herbal medicine) for the treatment of primary dysmenorrhea: a systematic review and meta-analysis. Arch Gynecol Obstet 2017; 296(4): 679-689.

9. He DY, Dai SM. Anti-inflammatory and immunomodulatory effects of paeonia lactiflora pall. a traditional chinese herbal medicine. Front Pharmacol 2011; 2: 10.

10. Sun L, Liu L, Zong S, Wang Z, Zhou J, Xu Z, Ding G, Xiao W, Kou J. Traditional Chinese medicine Guizhi Fuling capsule used for therapy of dysmenorrhea via attenuating uterus contraction. J Ethnopharmacol 2016; 191: 273-279.

11. Lee JH, Kim DH, Song WK, Oh MK, Ko DK. Label-free imaging and quantitative chemical analysis of
Alzheimer's disease brain samples with multimodal multiphoton nonlinear optical microspectroscopy. J Biomed Opt 2015; 20(5): 56013.

12. Luczak M, Suszynska-Zajczyk J, Marczak L, Formanowicz D, Pawliczak E, Wanic-Kossowska M, Stobiecki M. Label-Free Quantitative Proteomics Reveals Differences in Molecular Mechanism of Atherosclerosis Related and Non-Related to Chronic Kidney Disease. Int J Mol Sci 2016; 17(5).

13. Bostanci N, Selevsek N, Wolski W, Grossmann J, Bao K, Wahlander A, Trachsel C, Schlapbach R, Öztürk vÖ, Afacan $B$ et al. Targeted Proteomics Guided by Labelfree Quantitative Proteome Analysis in Saliva Reveal Transition Signatures from Health to Periodontal Disease. Mol Cell Proteomics 2018; 17(7): 1392-1409.

14. Zhang $Y$, Zhan $C$, Chen $G$, Sun J. Labelfree quantitative proteomics and bioinformatics analyses of alcoholic liver disease in a chronic and binge mouse model. Mol Med Rep 2018; 18(2): 2079-2087.

15. Patel VJ, Thalassinos K, Slade SE, Connolly JB, Crombie A, Murrell JC, Scrivens JH. A comparison of labeling and label-free mass spectrometry-based proteomics approaches. J Proteome Res 2009; 8(7): 3752-3759.

16. Bantscheff $M$, Lemeer $S$, Savitski MM, Kuster $B$. Quantitative mass spectrometry in proteomics: critical review update from 2007 to the present. Anal Bioanal Chem 2012; 404(4): 939-965.

17. Powell AM, Chan WY, Alvin P, Litt IF. Menstrual-PGF2 alpha, PGE2 and TXA2 in normal and dysmenorrheic women and their temporal relationship to dysmenorrhea. Prostaglandins 1985; 29(2): 273-290. 